\title{
Avaliação do Índice de Danos Permanentes Através de SLICC/ACR-DI em Pacientes com Mais de Cinco Anos de Diagnóstico de Lúpus Eritematoso Sistêmico(*)
}

\section{Cumulative Organ Damage Evaluation Using SLICC/ACR-DI in Patients with More Than Five Years of Systemic Lupus Erythematosus}

\author{
Elaine Marcelina Claudio Sella ${ }^{(1)}$ e Emilia Inoue Sato ${ }^{(2)}$
}

\section{RESUMO}

Objetivo: avaliar a freqüência dos diferentes tipos de lesões irreversíveis que ocorrem em pacientes com lúpus eritematoso sistêmico (LES) com mais de cinco anos de doença. Métodos: noventa pacientes do sexo feminino com diagnóstico de LES há mais de cinco anos foram avaliadas quanto às lesões irreversíveis em órgãos e/ou sistemas, através da aplicação do índice de dano do Systemic Lupus International Collaborating Clinics/American College of Rheumatology (SLICC/ACR-DI). Resultados: a média de idade das pacientes foi $38 \pm 10$ anos (60\% da cor branca) e o tempo de diagnóstico de LES foi $128 \pm 59$ meses; a média do número de critérios do ACR foi $7 \pm 1$ e a média dos escores do SLICC/ACR-DI foi $2 \pm 2$. Setenta e dois por cento das pacientes apresentaram SLICC/ ACR-DI $\geq 1$. Houve tendência à associação estatística entre idade atual mais avançada e presença de lesões permanentes $(p=0,06)$. Não observamos associação estatisticamente significante entre idade na época do diagnóstico de LES, tempo de doença ou número de critérios do ACR e presença de danos permanentes $(p>0,05)$. Foi encontrada associação significante entre lesão discóide e presença de dano irreversível $(p=0,049)$ e entre presença de anticorpos antidsDNA e lesão irreversível $(\mathrm{p}=0,013)$. Quanto aos tipos de danos permanentes, relatamos alterações cardiovasculares em 21\%, dermatológicas em 21\%, musculoesqueléticas em 17\%, gonadais em $16 \%$, neuropsiquiátricas em $13 \%$, oculares em $12 \%$, renais em $12 \%$, vasculares periféricas em $10 \%$, pulmonares em $6 \%$, endócrinas em $6 \%$ e malignidades em 3\% dos casos. Não houve associação estatisticamente significante entre tempo de uso de prednisona e presença de alteração irreversível $(p=0,725)$, entretanto, houve associação estatisticamente significante entre maior dose cumulativa de prednisona e danos permanentes $(\mathrm{p}=0,013)$. Houve tendência à

\begin{abstract}
Objective: to evaluate the frequency of different types of irreversible lesions in systemic lupus erythematosus (SLE) patients with more than five years of disease. Methods: ninety female SLE patients with more than five years of disease were evaluated concerning the irreversible damage score using Systemic Lupus International Collaborating Clinics/American College of Rheumatology Damage Index for systemic lupus erythematosus (SLICC/ACR/DI). Results: patients mean age was $38 \pm 10$ years old $(60 \%$ white). The average time after diagnosis was $128 \pm 59$ months, the mean number of ACR criteria was $7 \pm 1$, and the mean SLICC/ACR-DI score was 2 \pm 2 . Seventy-two percent of patients presented SLICC/ACRDI score $\geq 1$. We found a tendency to association between actual older age and presence of damage $(p=0.06)$. We did not find association between age at time of SLE diagnosis, disease duration or number of ACR criteria and presence of irreversible damage $(p>0.05)$, but we found a significant association between discoid lesion and presence of damage $(p=0.049)$ as well as between positive double strand antiDNA antibody and damage $(p=0.013)$. Concerning the type of irreversible lesions, we found cardiovascular damage in $21 \%$, dermatological lesions in 21\%, musculoskeletal lesions in $17 \%$, gonadal lesions in 16\%, neuropsychiatric damage in $13 \%$, eye lesions in $12 \%$, renal lesions in $12 \%$, peripheral vascular lesions in $10 \%$, pulmonary damage in $6 \%$, endocrinological damage in $6 \%$ and malignances in 3\%. There was no significant association between duration of prednisone use and damage $(p=0.725)$, however we found a significant association between prednisone cumulative dose and presence
\end{abstract}

\footnotetext{
* Disciplina de reumatologia da Universidade Federal de São Paulo/Escola Paulista de Medicina (Unifesp/EPM). Recebido em 23/4/2003. Aprovado, após revisão, em 30/9/2003.

1. Mestre em reumatologia pela Unifesp/EPM.

2. Professora titular da Unifesp/EPM.
}

Endereço para correspondência: Elaine Marcelina Claudio Sella. Disciplina de reumatologia da Universidade Federal de São Paulo/ Escola Paulista de Medicina (Unifesp/EPM). Rua Botucatu, 740, 3. ${ }^{\circ}$ andar, V. Clementino, CEP 04023-900, São Paulo, SP. E-mail: emiliasato@reumato.epm.br 
associação estatística entre menor tempo de uso de agentes antimaláricos e danos permanentes $(\mathrm{p}=0,068)$. Conclusões: lesões irreversíveis foram encontradas em $72 \%$ das pacientes com diagnóstico de LES há mais de cinco anos. As lesões mais prevalentes foram os acometimentos cardiovasculares, dermatológicos, musculoesqueléticos e gonadais. Dose cumulativa total de prednisona foi a variável que apresentou associação significante com presença de lesões irreversíveis em pacientes com LES, sugerindo a importância de estratégias para poupar corticoesteróides.

Palavras-chave: lúpus eritematoso sistêmico, prognóstico, morbidade, índice de dano, SLICC/ACR-DI.

\section{INTRODUÇÃO}

Em virtude do diagnóstico precoce, do avanço das técnicas laboratoriais e de novas opções terapêuticas, o prognóstico de lúpus eritematoso sistêmico (LES) tem melhorado nas últimas décadas ${ }^{(1-5)}$.

Vários autores têm avaliado os possíveis fatores relacionados ao prognóstico dos pacientes com LES e os resultados mostramse conflitantes. Muitos foram os fatores associados ao pior prognóstico, como idade mais avançada na ocasião do diagnóstico de $\operatorname{LES}^{(6,7)}$, raça negra ${ }^{(6,8,9)}$, sexo masculino ${ }^{(3,10)}$, nível sócio-econômico ${ }^{(5,9)}$, doença renal ${ }^{(3,7,11-13)}$, envolvimento pulmonar ${ }^{(7,13)}$, envolvimento cárdio-pulmonar ${ }^{(13)}$, comprometimento do sistema nervoso central e/ou periférico ${ }^{(12)}$, trombocitopenia ${ }^{(3,6,7,11)}$, tromboembolismo ${ }^{(11)}$, vasculite digital $^{(12)}$, hipertensão arterial sistêmica $(\mathrm{HAS})^{(12,14)}$, maior atividade de doença pelo escore do disease activity index for systemic lupus erythematosus (SLE-DAI) $>10^{(11)}$ e SLE-DAI $\geq 20^{(7)}$ na apresentação da doença e freqüência de exacerbações do LES após o seu diagnóstico ${ }^{(15)}$.

Por ser o LES doença polimórfica e crônica, caracterizada por amplo espectro de manifestações clínicas com períodos de remissões e exacerbações, torna-se imprescindível a existência de uma medida clínica prognóstica na evolução da doença. Nesse sentido, a análise periódica das injúrias irreversíveis decorrentes do LES pode ser avaliada por instrumento validado, através dos critérios propostos pelo Systemic Lupus International Collaborating Clinics/ American College of Rheumatology damage index for systemic lupus erythematosus (SLICC/ACR-DI). Este índice tem valor prognóstico e define os danos permanentes provocados pelo LES per se, pelas medidas terapêuticas e/ou co-morbidades associadas. Por definição, dano permanente foi considerado como alteração não relacionada com a of damage $(p=0.013)$. We found a tendency to association between lower duration of antimalarial drugs and irreversible damage $(p=0.068)$. Conclusions: irreversible lesions were found in $72 \%$ of SLE patients with more than five years of disease. The more prevalent lesions were cardiovascular, dermatological, musculoskeletal and gonadal. The cumulative dose of prednisone showed significant association with presence of damage, suggesting the importance of spare steroid strategies.

Keywords: systemic lupus erythematosus, prognosis, morbidity, damage index, SLICC/ACR-DI.

inflamação ativa no momento e sim aquela presente por, no mínimo, seis meses. Doze órgãos/sistemas são avaliados por escores: ocular (0-2), neuropsiquiátrico (0-6), renal (0-3), pulmonar (0-5), cardiovascular (0-6), vascular periférico (0-5), gastrointestinal (0-6), musculoesquelético $(0-7)$, cutâneo (0-3), endócrino (diabetes melito)(0-1), gonadal (0-1) e malignidades (0-2), no total máximo teórico de 47 pontos (questionário no final do artigo) ${ }^{(16-19)}$.

Estudando as causas de hospitalização de pacientes com LES em unidade de emergência, maior escore de SLICC/ ACR-DI foi a variável associada à hospitalização ${ }^{(20)}$. Pacientes com danos precoces observados já na primeira avaliação clínica apresentaram menor probabilidade de sobrevida após dez anos de seguimento, quando comparados com pacientes sem alterações permanentes precoces ${ }^{(21)}$.

Maiores índices de SLICC/ACR-DI foram associados com baixo status sócio-econômico e baixo nível educacional ${ }^{(22)}$, idade mais avançada na época do diagnóstico de $\operatorname{LES}^{(23,24)}$, idade maior que $50 \operatorname{anos}^{(25)}$, maior duração da doença ${ }^{(23,24,26)}$, maior atividade do LES na época do diagnóstico ${ }^{(23,24)}$ e maiores escores de SLE-DAI durante o período de estudo ${ }^{(25,27)}$. Houve correlação entre altos índices do SLICC/ACR-DI e persistência de atividade de doença com SLE-DAI $>10$. A sobrevida foi menor em pacientes com escores do SLICC/ ACR-DI $>2$ e de SLE-DAI>10 na última avaliação, confirmando o valor prognóstico do escore de danos permanentes do $\operatorname{LES}^{(27)}$.

O objetivo do presente estudo foi avaliar os escores do SLICC/ACR-DI em pacientes com diagnóstico de LES há mais de cinco anos e definir a freqüência dos diferentes tipos de danos permanentes. Secundariamente, avaliar a possível associação entre presença de lesões permanentes e idade, critérios de classificação do ACR e uso de medicações como prednisona e cloroquina. 


\section{PACIENTES E MÉTODOS}

No período entre 2000 e 2001 foi realizada revisão dos prontuários de pacientes com diagnóstico de LES em seguimento regular no ambulatório de doenças reumáticas autoimunes do serviço de reumatologia do Hospital São Paulo - Universidade Federal de São Paulo/Escola Paulista de Medicina (Unifesp/EPM).

Foram incluídos pacientes com quatro ou mais critérios para a classificação de LES, de acordo com o $\mathrm{ACR}^{(28,29)}$, do sexo feminino e com tempo de diagnóstico da doença superior a cinco anos. O projeto de estudo foi aprovado pelo Comitê de Ética e Pesquisa Médica da Unifesp/EPM e as pacientes assinaram termo de consentimento pós-informação.

As participantes foram submetidas ao exame físico completo e os seguintes dados foram obtidos através dos prontuários: tempo de diagnóstico do LES, critérios diagnósticos e outras manifestações clínicas da doença, dados laboratoriais gerais e imunológicos e lesões irreversíveis em órgãos e/ou sistemas.

Para o cálculo do tempo de uso e dose cumulativa de prednisona e tempo de uso de agentes antimaláricos foram consideradas apenas as pacientes que tiveram diagnóstico de LES no ambulatório da Unifesp/EPM e permaneceram, desde então, em acompanhamento contínuo a intervalos máximos de quatro meses.

A avaliação psiquiátrica foi obtida pelas informações dos prontuários, não sendo aplicado questionário específico para o estudo da função cognitiva.

\section{ANÁLISE ESTATÍSTICA}

Os testes de qui-quadrado e qui-quadrado de Pearson foram utilizados para verificar a relação de dependência entre as variáveis qualitativas. O teste exato de Fisher foi empregado quando a amostra foi $<30$ ou as freqüências esperadas foram $<5$. O teste de Mann-Whitney foi usado para comparar os subgrupos de pacientes com relação às variáveis quantitativas ${ }^{(30)}$.

Foram considerados significantes os resultados com nível descritivo $(\mathrm{p})<0,05$ e que foram assinalados com um asterisco $\left({ }^{*}\right)$. A tendência à significância estatística foi admitida nos casos de valores de $\mathrm{p} \geq 0,05$ e $<0,10$.

\section{RESULTADOS}

Participaram deste estudo 90 pacientes com LES, com média de idade de $38 \pm 10$ anos, $60 \%$ da cor branca, média da idade por ocasião do diagnóstico do LES de $27 \pm 9$ anos e média do tempo de diagnóstico de LES de $128 \pm 59$ meses
(Tabela 1). O escore do SLICC/ACR-DI foi $2 \pm 2$, com variação de 0 a 6 .

Os sintomas constitucionais mais comumente relacionados ao LES, ao longo de sua evolução, foram alopecia em 79 pacientes (88\%), febre em 61 (68\%), fadiga em 58 (64\%) e perda de peso corpóreo em 49 (54\%). O número de critérios do ACR, na época do estudo, foi $7 \pm 1$ (mediana 7 , variando de 4 a 10). As seguintes freqüências dos critérios do ACR foram observadas: eritema malar em $91 \%$ dos casos, lesão discóide em $23 \%$, fotossensibilidade em $90 \%$, vasculite de mucosa em 38\%, acometimento articular em $88 \%$, serosite em 37\%, acometimento renal em 49\%, acometimento neurológico em $11 \%$, acometimento hematológico em $87 \%$, alterações imunológicas em $82 \%$ e anticorpos antinucleares em $99 \%$ das pacientes.

Vasculite digital foi descrita em prontuários de 46 pacientes $(51 \%)$. Outras vasculites, que incluíram úlceras não mucosas ou digitais, vasculites viscerais e do sistema nervoso central comprovadas por exames subsidiários, foram relatadas em $16(18 \%)$. Trombose venosa foi definida em $6(7 \%)$ e trombose arterial em 3 (3\%). Anticorpos anticardiolipina foram positivos em 34 pacientes (38\%).

TABELA 1

Medidas de TENDÊNCIA CENTRAL E DE DisPersão de IDADE ATUAL, IDADE DAS PACIENTES NA ÉPOCA DO DIAGNÓSTICO E TEMPO DE LES

NOS SUBGRUPOS DE PACIENTES COM E SEM DANOS PERMANENTES

\begin{tabular}{|c|c|c|c|c|}
\hline Variáveis & 90 LES & $\begin{array}{c}\text { SLICC / } \\
\text { ACR-DI = } 0 \\
N=25\end{array}$ & $\begin{array}{c}\text { SLICC / } \\
\text { ACR-DI } \geq 1 \\
N=65\end{array}$ & $\mathbf{P}$ \\
\hline \multicolumn{5}{|l|}{$\begin{array}{l}\text { Idade } \\
\text { atual (anos) }\end{array}$} \\
\hline$M D \pm D P$ & $38 \pm 10$ & $35 \pm 8$ & $39 \pm 10$ & \\
\hline Mediana & 39 & 35 & 41 & 0,06 \\
\hline Mín. / máx. & $20 / 55$ & $22 / 50$ & $20 / 55$ & \\
\hline \multicolumn{5}{|c|}{$\begin{array}{l}\text { Idade } \\
\text { diagnóstico (anos) }\end{array}$} \\
\hline $\mathrm{MD} \pm \mathrm{DP}$ & $27 \pm 9$ & $25 \pm 7$ & $28 \pm 9$ & \\
\hline Mediana & 26 & 25 & 27 & 0,353 \\
\hline Mín. / máx. & $12 / 45$ & $12 / 38$ & $13 / 45$ & \\
\hline \multicolumn{5}{|c|}{$\begin{array}{l}\text { Tempo } \\
\text { de LES (meses) }\end{array}$} \\
\hline$M D \pm D P$ & $128 \pm 59$ & $113 \pm 40$ & $134 \pm 64$ & \\
\hline Mediana & 112 & 102 & 120 & 0,319 \\
\hline Mín. / máx. & $61 / 312$ & $67 / 204$ & $61 / 312$ & \\
\hline \multicolumn{5}{|c|}{ Teste de Mann-Whitney } \\
\hline $\begin{array}{l}\text { MD } \pm \text { DP } \\
\text { Mín. / máx. } \\
\text { P } \\
\text { SLICC/ACR-DI }\end{array}$ & $\begin{array}{l}\text { Média } \pm \text { desvio-padrã } \\
\text { Valores mínimo e má } \\
\text { Nivel descritivo } \\
\text { Systemic Lupus Intern } \\
\text { College of Rheumatoll }\end{array}$ & $\begin{array}{l}\text { mo das variáveis } \\
\text { tional Collaboratir } \\
\text { y damage index }\end{array}$ & Clinics / A & \\
\hline
\end{tabular}


Houve tendência à associação estatística entre idade atual mais avançada e presença de danos permanentes $(p=0,06)$. Não observamos associação estatisticamente significante entre idade na época do diagnóstico de LES e presença de lesões permanentes $(p=0,353)$. Não houve associação estatisticamente significante entre tempo de doença e presença de danos permanentes $(p=0,319)$ (Tabela 1).

Cinqüenta e duas pacientes (58\%) tinham diagnóstico de LES entre 61 e 120 meses, 24 (27\%) entre 121 e 180 meses e 14 (15\%) com LES há mais de 180 meses. Não houve diferença estatisticamente significante com relação à presença de injúrias permanentes nesses subgrupos de pacientes $(p=0,466)$. Vinte e cinco pacientes $(28 \%)$ não apresentaram lesões permanentes e 65 (72\%) tinham escores de SLICC/ACR-DI $\geq 1$ (Tabela 2).

TABELA 2

Escores De SLICC/ACR-DI NAS 90 PACIENTES

\begin{tabular}{lc}
\hline Escores do SLICC/ACR-DI & Freqüências (\%) \\
\hline SLICC/ACR-DI $=1$ & $25(39)$ \\
SLICC/ACR-DI $=2$ & $17(26)$ \\
SLICC/ACR-DI $=3$ & $9(14)$ \\
SLICC/ACR-DI $=4$ & $8(12)$ \\
SLICC/ACR-DI $=5$ & $4(6)$ \\
SLICC/ACR-DI $=6$ & $2(3)$ \\
\hline
\end{tabular}

SLICC/ACR-DI Systemic Lupus International Collaborating Clinics / American College of Rheumatology damage index

Não foi observada associação estatisticamente significante entre número de critérios do ACR e presença de danos permanentes $(p=0,308)$. Lesão discóide e anticorpos anti-dsDNA foram os critérios diagnósticos que mostraram associação estatisticamente significante com o índice de danos permanentes $(\mathrm{p}=0,049$ e 0,013, respectivamente). Houve tendência à associação estatística entre trombocitopenia e presença de danos permanentes $(\mathrm{p}=0,07)$.

Os danos mais freqüentemente observados foram: alterações cardiovasculares em $21 \%$, dermatológicas em $21 \%$, musculoesqueléticas em 17\%, gonadais em 16\%, neuropsiquiátricas em $13 \%$, oculares em $12 \%$, renais em $12 \%$, vasculares periféricas em $10 \%$, pulmonares em $6 \%$, endócrinas em $6 \%$ e malignidades em $3 \%$ dos casos. Os danos permanentes em órgãos e/ou sistemas avaliados pelo SLICC/ ACR-DI são apresentados na Tabela 3.
TABELA 3

FREQÜÊNCIAS E PORCENTAGENS DE DANOS PERMANENTES PELO SLICC/ACR-DI DAS 90 PACIENTES

\begin{tabular}{|c|c|}
\hline Variáveis -SLICC/ACR-DI & Freqüências (\%) \\
\hline $\begin{array}{l}\text { Ocular } \\
\text { Catarata }\end{array}$ & $\begin{array}{c}11(12) \\
7(8)\end{array}$ \\
\hline Retinopatia & $3(3)$ \\
\hline Ceratite com deficiência visual & $2(2)$ \\
\hline Neurite óptica & $1(1)$ \\
\hline $\begin{array}{l}\text { Neuropsiquiátrico } \\
\text { Disfunção cognitiva }\end{array}$ & $\begin{array}{c}12(13) \\
2(2)\end{array}$ \\
\hline Psicose maior & $1(1)$ \\
\hline Anticonvulsivantes por mais de 6 meses & $4(4)$ \\
\hline Acidente vascular cerebral & $2(2)$ \\
\hline Neuropatia craniana & $2(2)$ \\
\hline Neuropatia periférica & $2(2)$ \\
\hline Neuropatia múltipla & $1(1)$ \\
\hline $\begin{array}{l}\text { Renal } \\
\quad \text { Proteinúria } \geq 3,5 \mathrm{~g} / 24 \text { horas }\end{array}$ & $\begin{array}{l}11(12) \\
10(11)\end{array}$ \\
\hline Insuficiência renal terminal - diálise & $1(1)$ \\
\hline $\begin{array}{l}\text { Pulmonar } \\
\quad \text { Hipertensão pulmonar } \\
\text { Fibrose pulmonar }\end{array}$ & $\begin{array}{l}5(6) \\
1(1) \\
5(6)\end{array}$ \\
\hline $\begin{array}{l}\text { Cardiovascular } \\
\text { Angina }\end{array}$ & $\begin{array}{c}19(21) \\
8(9)\end{array}$ \\
\hline Infarto agudo do miocárdio & $1(1)$ \\
\hline Miocardiopatia & $12(13)$ \\
\hline Doença valvular & $4(4)$ \\
\hline Pericardite por 6 meses & $1(1)$ \\
\hline $\begin{array}{l}\text { Vascular periférico } \\
\text { Perda de polpa tecidual }\end{array}$ & $\begin{array}{c}9(10) \\
6(7)\end{array}$ \\
\hline Estase venosa & $4(4)$ \\
\hline Gastrointestinal & 0 \\
\hline $\begin{array}{l}\text { Musculoesquelético } \\
\text { Atrofia muscular e fraqueza }\end{array}$ & $\begin{array}{c}15(17) \\
1(1)\end{array}$ \\
\hline Deformidade articular redutível ou erosiva & $7(8)$ \\
\hline Osteoporose com colapso vertebral & $2(2)$ \\
\hline Osteonecrose & $4(4)$ \\
\hline Osteomielite & $2(2)$ \\
\hline $\begin{array}{l}\text { Dermatológico } \\
\text { Alopecia crônica cicatricial }\end{array}$ & $\begin{array}{l}19(21) \\
10(11)\end{array}$ \\
\hline Cicatriz cutânea extensa & $7(8)$ \\
\hline Ulceração cutânea por mais de 6 meses & $5(6)$ \\
\hline Falência gonadal prematura & $14(16)$ \\
\hline Diabetes melito & $5(6)$ \\
\hline Malignidade & $3(3)$ \\
\hline Carcinoma epidermóide (colo uterino) & $1(1)$ \\
\hline Carcinoma basocelular pigmentado (pele) & $1(1)$ \\
\hline
\end{tabular}

SLICC/ACR-DI Systemic Lupus International Collaborating Clinics / American College of Rheumatology damage index 
Quarenta e oito pacientes tiveram seguimento clínico contínuo em nosso serviço. Todas as pacientes, em algum momento ao longo da doença, receberam esteróides e 44 pacientes fizeram uso de medicação antimalárica. Houve tendência à associação estatística entre menor tempo de uso de antimaláricos (em meses) e lesões permanentes $(\mathrm{p}=0,068)$. Quanto à corticoterapia, não houve associação estatisticamente significante entre tempo de uso de prednisona (em meses) e presença de alteração irreversível $(\mathrm{p}=0,725)$. Observamos, no entanto, associação estatisticamente significante entre maior dose cumulativa de prednisona (em gramas) e danos permanentes $(\mathrm{p}=0,013)$ (Tabela 4$)$.

\section{TABELA 4}

MEdidAs DE TENDÊNCIA CENTRAL E DE DISPERSÃO DE TEMPO DE USO DE ANTIMALÁRICOS E PREDNISONA E DOSE CUMULATIVA DE PREDNISONA NOS SUBGRUPOS DAS 48 PACIENTES COM E SEM DANOS PERMANENTES

\begin{tabular}{lcccc}
\hline Variáveis & $\mathbf{4 8}$ LES & $\begin{array}{c}\text { SLICC / } \\
\text { ACR-DI }=0 \\
\mathrm{~N}=14\end{array}$ & $\begin{array}{c}\text { SLICC / } \\
\text { ACR-DI } \geq 1 \\
\mathrm{~N}=\mathbf{3 4}\end{array}$ & $\mathbf{P}$ \\
\hline $\begin{array}{l}\text { Antimaláricos } \\
\text { (meses) } \\
\text { MD } \pm \text { DP }\end{array}$ & $33 \pm 33$ & $44 \pm 35$ & $28 \pm 32$ & \\
Mediana & 21 & 35 & 18 & 0,068 \\
Mín. / máx. & $0 / 132$ & $5 / 118$ & $0 / 132$ &
\end{tabular}

\section{Corticoterapia}

(meses)

$\begin{array}{lcccc}\text { MD } \pm \text { DP } & 98 \pm 50 & 89 \pm 31 & 102 \pm 56 & \\ \text { Mediana } & 88 & 88 & 88 & 0,725 \\ \text { Mín. / máx. } & 27 / 272 & 35 / 145 & 28 / 272 & \end{array}$

\begin{tabular}{lcccc}
$\begin{array}{l}\text { Dose cumulativa } \\
\text { PD }(g)\end{array}$ & & & & \\
MD \pm DP & $54 \pm 28$ & $38 \pm 17$ & $61 \pm 29$ & \\
Mediana & 45 & 34 & 57 & $0,013^{*}$ \\
Mín. / máx. & $11,99 /$ & $11,99 /$ & $15,45 /$ & \\
& 137,12 & 83,47 & 137,12 & \\
\hline
\end{tabular}

Teste de Mann-Whitney

$M D \pm D P \quad$ Média \pm desvio-padrão

${ }^{*} \mathrm{p}<0,05$ (significante)

Mín. / máx. Valores mínimo e máximo das variáveis

$\mathrm{p} \quad$ Nível descritivo

PD Prednisona

SLICC/ACR-DI Systemic Lupus International Collaborating Clinics / American

College of Rheumatology damage index

\section{DISCUSSÃO}

Como conseqüência da melhora da sobrevida, os pacientes com diagnóstico de LES têm apresentado consideráveis comorbidades e isto se deve às seqüelas de atividade da doença e aos efeitos colaterais do tratamento ${ }^{(31-33)}$. A introdução dos esteróides foi um dos fatores importantes para a melhora da expectativa de vida desses pacientes. No entanto, a corticoterapia tem sido implicada como fator de risco para doença vascular aterosclerótica, miopatia, osteoporose, osteonecrose e catarata em $\operatorname{LES}^{(31,34,35)}$.

Observamos que pacientes com diagnóstico de LES há mais de cinco anos apresentaram escore de SLICC/ACRDI $2 \pm 2$. Rivest et al. ${ }^{(24)}$ avaliaram pacientes com duração média de LES de 3,8 (desvio-padrão 2,2) anos e o escore de danos permanentes relatado foi $1,5 \pm 1,8$. Molad et al. ${ }^{(25)}$ encontraram média de escore de SLICC/ACR-DI de $0,17 \pm 0,64$ na primeira avaliação e de $1,64 \pm 2,1$ após acompanhamento de $46 \pm 37$ meses, com diferença estatisticamente significante.

Setenta e dois por cento de nossas pacientes apresentaram algum dano irreversível, maior porcentagem quando comparada com relatos de literatura, que têm variado de $9,4 \%$ a $56 \% \%^{(21,25-27,36-38)}$. Esta diferença provavelmente devese ao fato de incluirmos neste estudo pacientes com mais de cinco anos de LES.

Como observado em estudos anteriores ${ }^{(27,36)}$, não foi encontrada associação entre idade por ocasião do estudo ou do diagnóstico de LES e presença de danos permanentes. Em outro trabalho, idade mais avançada na época do diagnóstico de LES mostrou correlação com presença de danos cardiovascular, musculoesquelético, gastrointestinal, ocular e pulmonar e maior tempo de diagnóstico de doença correlacionouse com maiores danos renal e cardiovascular. Maior atividade de LES na época do diagnóstico correlacionou-se com maiores danos renal, musculoesquelético e pulmonar ${ }^{(24)}$.

Como relatado em estudo anterior ${ }^{(36)}$, a presença de lesão discóide foi preditiva de dano irreversível. No presente estudo houve associação significante entre presença de anticorpos anti-dsDNA e índice de danos permanentes. Esta associação não havia sido estudada em outros trabalhos.

Relatamos as maiores freqüências de danos permanentes cardiovasculares (21\%), dermatológicos (21\%), musculoesqueléticos (17\%) e gonadal (16\%). De um modo geral, estas freqüências são maiores que as referidas na literatura, que mostra alterações musculoesqueléticas irreversíveis em 2,7\% a $18,5 \%^{(24,25,36)}$, danos cardiovasculares em $1,6 \%$ a $17,2 \%$, dermatológicos em $4 \%$ a $12,5 \%$ e falência gonadal prematura em $2 \%$ a $11 \%$ dos pacientes ${ }^{(24-26,36)}$. Provavelmente isto se deve ao fato de termos estudado pacientes com maior tempo de doença.

Injúrias neuropsiquiátricas permanentes foram observadas em $13 \%$ das pacientes, porcentagem esta dentro da variação referida pela literatura $(6,6 \% \text { a } 32,4 \%)^{(24-26,36)}$. Danos oculares 
estiveram presentes em $12 \%$ de nossas pacientes e os autores os relatam em $1,1 \%$ a $18,5 \%{ }^{(24-26,36)}$.

Observamos danos renais em $12 \%$, em concordância com as freqüências relatadas de $8,2 \%$ a $11,9 \% \%^{(24-26,36)}$. Num estudo, menor idade no diagnóstico de LES e trombocitopenia foram os fatores de risco independentes para injúria renal ${ }^{(36)}$. Neste estudo, houve tendência à associação estatística entre trombocitopenia e danos permanentes. Em outro estudo, escore de dano renal permanente foi relatado como preditivo de doença renal terminal ${ }^{(39)}$.

Alterações vasculares periféricas foram observadas em 10\% das pacientes. Outros autores relataram freqüências entre 1,1\% e 9,3\% ${ }^{(24-26,36)}$. Danos permanentes pulmonares e endócrinos estiveram presentes em $6 \%$ dos casos. Há relatos de alterações pulmonares em $1,1 \%$ a $12,6 \%$ dos pacientes e de diabetes melito em $0,5 \%$ a 5,3\%(24-26,36). Escore de dano pulmonar foi definido como preditivo de óbito em dez anos ${ }^{(39)}$.

Observamos malignidade em 3\% das pacientes, frequiência similar à relatada previamente (até 4,6\%) $)^{(24-26,36)}$.

O tempo de uso de agentes antimaláricos e de prednisona e a dose cumulativa aproximada de prednisona foram calculados apenas para as pacientes com prontuários mais completos e em seguimento contínuo no nosso serviço desde o diagnóstico de LES. A seleção de apenas 48 das 90 pacientes para tal avaliação teve por finalidade minimizar os erros inerentes a esses cálculos através da revisão dos prontuários médicos.

Neste estudo, houve tendência à associação estatística entre menor tempo de uso de antimaláricos e presença de danos permanentes. Molad et al. ${ }^{(25)}$ encontraram associação significante entre terapia com hidroxicloroquina e menor escore de SLICC/ACR-DI, demonstrando o efeito protetor das drogas antimaláricas no LES.
Observamos associação estatisticamente significante entre maior dose cumulativa de prednisona e presença de alterações permanentes. Os trabalhos da literatura trazem dados conflitantes. Molad et al. ${ }^{(25)}$ relataram associação estatisticamente significante entre dose diária de prednisona e maior escore de SLICC/ACR-DI, sugerindo que os efeitos adversos dos esteróides provoquem danos permanentes. Rahman et al. ${ }^{(21)}$ encontraram maior dose média cumulativa de corticoesteróide no primeiro ano de avaliação dos pacientes no subgrupo com danos permanentes que nos pacientes sem danos precoces. Por outro lado, Nossent ${ }^{(27)}$ não observou correlação entre dosagem diária de esteróide ou dose cumulativa da droga e escore do SLICC/ACR-DI.

No seguimento clínico dos pacientes com LES são imperativos o reconhecimento dos períodos de exacerbação e a distinção entre atividade de doença e os resultados de efeitos cumulativos de destruição orgânica previamente existente $^{(40)}$. Nesse sentido, o SLICC/ACR-DI poderia refletir o impacto da persistência de atividade de doença e/ou expressar os efeitos cumulativos de esteróides ${ }^{(18)}$.

Este estudo mostrou que $72 \%$ das pacientes com diagnóstico de LES há mais de cinco anos apresentaram lesões irreversíveis. As maiores freqüências observadas foram de acometimentos cardiovasculares, dermatológicos, musculoesqueléticos e gonadais. A dose cumulativa de prednisona foi importante variável associada à presença de lesões irreversíveis em pacientes com LES, sugerindo a relevância das estratégias para poupar corticoesteróides. Observamos, também, tendência à associação entre menor tempo de uso de agentes antimaláricos e maior escore do SLICC/ ACR-DI, sinalizando um possível efeito protetor das drogas antimaláricas.

DeFINIÇÃo DOS ESCORES DO SLICC/ACR-DI ${ }^{(16,17)}$

\begin{tabular}{|c|c|}
\hline ESCORE & DEFINIÇÃO \\
\hline 1 & Catarata em qualquer olho, primária ou secundária à corticoterapia, documentada por oftalmoscopia. \\
\hline \multirow[t]{2}{*}{1} & Atrofia óptica documentada por exame oftalmoscópico. \\
\hline & Neuropsiquiátrico \\
\hline 1 & $\begin{array}{l}\text { Psicose maior definida por distúrbios graves da percepção da realidade caracterizados por delírios, alucinações auditivas ou } \\
\text { visuais, incoerência, perda de associação de idéias, empobrecimento de idéias, pensamento ilógico, comportamento bizarro, } \\
\text { desorganizado ou catatônico. }\end{array}$ \\
\hline 1 & Convulsões caracterizadas por movimentos tônicos e clônicos, requerendo terapia anticonvulsivante por mais de seis meses. \\
\hline $1(2)$ & $\begin{array}{l}\text { Acidente vascular cerebral que resulte em achados focais como paresia e fraqueza ou ressecção cirúrgica por outras causas, exceto } \\
\text { malignidade (escore } 2 \text { se mais de um evento). }\end{array}$ \\
\hline
\end{tabular}




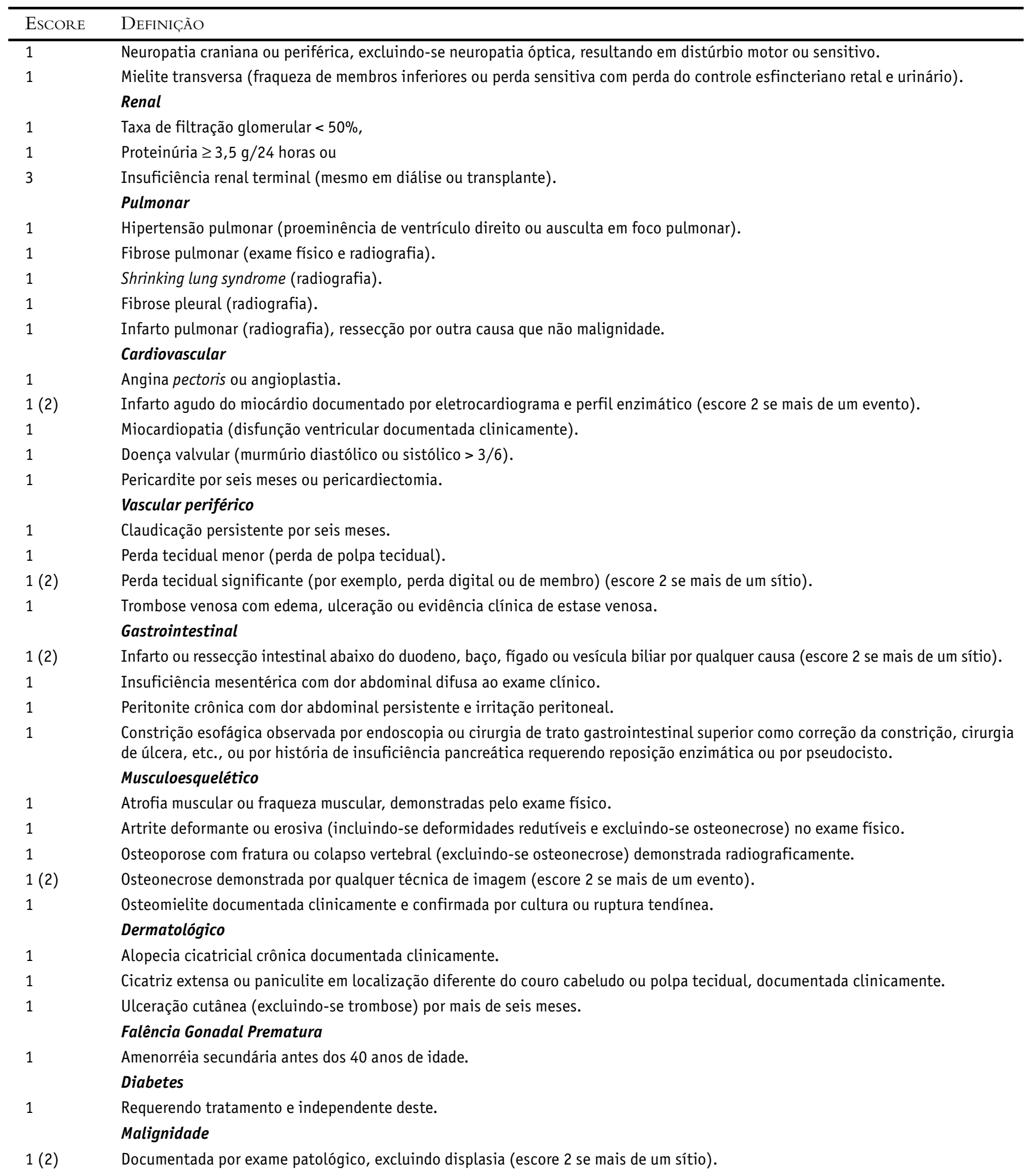

OBSERVAÇÃO - Considera-se dano a alteração não reversivel e não relacionada com a inflamação ativa ocorrida desde o início do LES. A avaliação é feita por médico e a alteração deve estar presente por pelo menos seis meses. Episódios repetidos devem ocorrer em intervalo de pelo menos seis meses para obterem escore 2. A mesma lesão não pode ser considerada duas vezes. 0 dano global do SLICC/ACR-DI descreve o escore total resultante do próprio LES ou de qualquer outro processo patológico como aterosclerose, hipercoagulabilidade, HAS, terapêutica do LES e outras co-morbidades. 


\section{REFERÊNCIAS}

1. Dubois EL, Wierzchowiecki M, Cox MB, Weiner JM: Duration and death in systemic lupus erythematosus - an analysis of 249 cases. JAMA 227: 1399-402, 1974.

2. Urowitz MB, Bookman AAM, Koehler BE, Gordon DA, Smythe HA, Ogryzlo MA: The bimodal mortality pattern of systemic lupus erythematosus. Am J Med 60: 221-5, 1976.

3. Pistiner M, Wallace DJ, Nessim S, Metzger AL, Klinenberg JR: Lupus erythematosus in the 1980s - a survey of 570 patients. Semin Arthritis Rheum 21: 55-64, 1991

4. Abu-Shakra M, Urowitz MB, Gladman DD, Gough J: Mortality studies in systemic lupus erythematosus - results from a single center: I. causes of death. J Rheumatol 22: 1259-64, 1995.

5. Ward MM, Pyun E, Studenski S: Long-term survival in systemic lupus erythematosus - patient characteristics associated with poorer outcomes. Arthritis Rheum 38: 274-83, 1995.

6. Reveille JD, Bartolucci A, Alarcón GS: Prognosis in systemic lupus erythematosus - negative impact of increasing age at onset, black race, and thrombocytopenia, as well as causes of death. Arthritis Rheum 33: 37-48, 1990.

7. Abu-Shakra M, Urowitz MB, Gladman DD, Gough J: Mortality studies in systemic lupus erythematosus - results from a single center: II. predictor variables for mortality. J Rheumatol 22: 1265-70, 1995.

8. Ginzler EM, Diamond HS, Weiner M, et al: A multicenter study of outcome in systemic lupus erythematosus - I. entry variables as predictors of prognosis. Arthritis Rheum 25: 601-11, 1982.

9. Studenski S, Allen NB, Caldwell DS, Rice JR, Polisson RP: Survival in systemic lupus erythematosus - a multivariate analysis of demographic factors. Arthritis Rheum 30: 1326-32, 1987.

10. Wallace DJ, Podell T, Weiner J, Klinenberg JR, Forouzesh S, Dubois EL: Systemic lupus erythematosus - survival pattern: experience with 609 patients. JAMA 245: 934-8, 1981.

11. Massardo L, Martínez ME, Jacobelli S, Villarroel L, Rosenberg H, Rivero S: Survival of chilean patients with systemic lupus erythematosus. Semin Arthritis Rheum 24: 1-11, 1994.

12. Sato EI, Vieira ALS, Pochini AC, et al: Fatores prognósticos no lúpus eritematoso sistêmico. Rev Bras Reumatol 35: 235-241, 1995.

13. Latorre LC: Análise da sobrevida em pacientes com lúpus eritematoso sistêmico. Tese de doutorado. Faculdade de Saúde Pública da Universidade de São Paulo, São Paulo, 1997.

14. Seleznick MJ, Fries JF: Variables associated with decreased survival in systemic lupus erythematosus. Semin Arthritis Rheum 21: 73-80, 1991.

15. Swaak AJG, Nossent JC, Bronsveld W, et al: Systemic lupus erythematosus - I. outcome and survival: dutch experience with 110 patients studied prospectively. Ann Rheum Dis 48: 447-54, 1989.

16. Gladman D, Ginzler E, Goldsmith C, et al: The development and initial validation of the systemic lupus international collaborating clinics/American College of Rheumatology damage index for systemic lupus erythematosus. Arthritis Rheum 39: 363-9, 1996.

17. Gladman $\mathrm{DD}$, Urowitz $\mathrm{MB}$, Goldsmith $\mathrm{CH}$, et al: The reliability of the systemic lupus international collaborating clinics/American College of Rheumatology damage index in patients with systemic lupus erythematosus. Arthritis Rheum 40: 809-13, 1997.

18. Gladman DD, Urowitz MB: The SLICC/ACR damage index progress report and experience in the field. Lupus 8: 632-7, 1999.

19. Gladman DD, Goldsmith $\mathrm{CH}$, Urowitz MB, et al: The systemic lupus international collaborating clinics/American College of Rheumatology damage index for systemic lupus erythematosus international comparison. J Rheumatol 27: 373-6, 2000.

20. Rojas-Serrano J, Cardiel MH: Lupus patients in an emergency unit. Causes of consultation, hospitalization and outcome. A cohort study. Lupus 9: 601-6, 2000.
21. Rahman P, Gladman DD, Urowitz MB, Hallet D, Tam LS: Early damage as measured by the SLICC/ACR damage index is a predictor of mortality in systemic lupus erythematosus. Lupus 10 : 93-6, 2001.

22. Lotstein DS, Ward MM, Bush TM, Lambert E, Van Vollenhoven R, Neuwelt M: Socioeconomic status and health in women with systemic lupus erythematosus. J Rheumatology 25: 1720-9, 1998.

23. Karlson EW, Daltroy LH, Lew RA, et al: The relationship of socioeconomic status, race, and modifiable risk factors to outcomes in patients with systemic lupus erythematosus. Arthritis Rheum 40: 47-56, 1997.

24. Rivest C, Lew RA, Welsing PMJ, et al: Association between clinical factors, socieconomic status, and organ damage in recent onset systemic lupus erythematosus. J Rheumatology 27: 680-4, 2000.

25. Molad Y, Gorshtein A, Wysenbeek AJ, et al: Protective effect of hydroxychloroquine in systemic lupus erythematosus. Prospective long-term study of an Israeli cohort. Lupus 11: 356-61, 2002.

26. Zonana-Nacach A, Camargo-Coronel A, Yáñez P, et al: Measurement of damage in 210 Mexican patients with systemic lupus erythematosus - relationship with disease duration. Lupus 7: 119-23, 1998.

27. Nossent JC: SLICC/ACR damage index in afro-caribbean patients with systemic lupus erythematosus - changes in and relationship to disease activity, corticosteroid therapy, and prognosis. J Rheumatol 25: 654-9, 1998.

28. Tan EM, Cohen AS, Fries JF, et al: The 1982 revised criteria for the classification of systemic lupus erythematosus. Arthritis Rheum 25: 1271-7, 1982.

29. Hochberg MC: Updating the American College of Rheumatology revised criteria for the classification of systemic lupus erythematosus. Arthritis Rheum 40: 1725, 1997 (letter).

30. Siegel J: Estatística não-paramétrica, Brasil, McGraw Hill do Brasil, 350, 1981.

31. Gladman DD, Urowitz MB: Morbidity in systemic lupus erythematosus. J Rheumatol 14 (suppl 13): 223-6, 1987.

32. American College of Rheumatology Ad Hoc Committee on systemic lupus erythematosus Guidelines: Guidelines for referral and management of systemic lupus erythematosus in adults. Arthritis Rheum 42: 1785-96, 1999.

33. Trager J, Ward MM: Mortality and causes of death in systemic lupus erythematosus. Curr Opin Rheumatol 13: 345-51, 2001.

34. Maxwell SRJ, Moots RJ, Kendall MJ: Corticosteroids: do they damage the cardiovascular system? Postgrad Med J 70: 863-70, 1994.

35. Urowitz MB, Gladman DD: How to improve morbidity and mortality in systemic lupus erythematosus. Rheumatology 39: 238-44, 2000.

36. Mok CC, Lee KW, Ho CTK, Lau CS, Wong RWS: A prospective study of survival and prognostic indicators of systemic lupus erythematosus in a southern Chinese population. Rheumatology 39: 399-406, 2000

37. Thumboo J, Lee HY, Fong KY, et al: Accuracy of medical record scoring of the SLICC/ACR damage index for systemic lupus erythematosus. Lupus 9: 358-62, 2000.

38. Stoll T, Sutcliffe N, Klaghofer R, Isenberg DA: Do present damage and health perception in patients with systemic lupus erythematosus predict extent of future damage? - a prospective study. Ann Rheum Dis 59: $832-5,2000$

39. Stoll T, Seifert B, Isenberg DA: SLICC/ACR damage index is valid, and renal and pulmonary organ scores are predictors of severe outcome in patients with systemic lupus erythematosus. Br J Rheumatol 35: 248-54, 1996.

40. Corzillius M, Liang MH: Monitoring patients with systemic lupus erythematosus. In: Lahita RG - Systemic lupus erythematosus, 3.a ed. San Diego, Academic Press (section IV, chapter 22), p 467-76, 1999. 